\title{
Arsenic Poisoning in Bangladesh, Impact on Health and Ecology and Mitigation Strategies
}

\author{
Muhammad Tariq Bashir \\ Department of Civil Engineering \\ Jouf University \\ Saudia Arabia
}

\author{
Raid Naif Alrowais \\ Department of Civil Engineering \\ Jouf University \\ Saudi Arabia
}

\author{
Muhammad Daniyal \\ Department of Civil Engineering, \\ Qurtuba University \\ Pakistan
}

\begin{abstract}
This paper delineates the impacts of arsenic on human and ecological health and subsequent treatments of arsenic related diseases in Bangladesh. Arsenic is a metalloid that is known for its toxicity and carcinogenicity. Arsenic causes arsenicosis and several carcinogenic diseases and other diseases. The high arsenic concentration in groundwater in the country is believed to be due to the over-exploitation of groundwater for irrigation purpose and domestic water supply, inadequate recharge of the aquifer and lack of water management in Bangladesh. Several tube-wells in Bangladesh have been found to contain arsenic in concentration that is beyond the permissible limit which is $0.05 \mathrm{mg} / \mathrm{l}(50 \mu \mathrm{g} / \mathrm{L})$. Up to 75 million people in Bangladesh are at risk of arsenic exposure. Arsenic affects crops and the food chain and can result to poor yield of crops. Some advanced technologies can also be leveraged to mitigate arsenic contamination. The government of Bangladesh has played a significant role in minimizing or eliminating arsenic contamination but it is not capable enough to deal with the problem. Remediation methods can be resorted to but most important would be to close down wells that have a concentration of arsenic beyond the permissible limit.
\end{abstract}

Keywords: Arsenic Contamination, Health Issues, Skin Lesions, Carcinogenicity, Chelation Therapy, Social Effects, Economic Implications

\section{INTRODUCTION}

Arsenic is a metalloid that prevail in the earth crust in combination with metals like iron, copper, sodium, calcium and so forth to form the salt $[1,2]$. This metalloid and its compound are known for their toxicity and carcinogenicity. Arsenic causes arsenicosis and several carcinogenic diseases and other diseases. Exposure to arsenic can occur via several means but especially through water, food, air, and so forth. The World Health Organization (WHO) drinking-water standard for arsenic concentration is 0.01 $\mathrm{mg} / \mathrm{L}(10 \mu \mathrm{g} / \mathrm{L})$, however, Bangladesh permissible limit is $0.05 \mathrm{mg} / \mathrm{l}(50 \mu \mathrm{g} / \mathrm{L})$. However, numerous studies have unveiled that various tube-wells (which is the main source of drinking water) in Bangladesh are contaminated with arsenic at a concentration that is beyond the Bangladesh permissible limit. Arsenic was first exposed in Bangladesh in 1993[2-5]. The high arsenic concentration in groundwater in the country is believed to be due to the over-exploitation of groundwater for irrigation purpose and domestic water supply, inadequate recharge of the aquifer and lack of water management in Bangladesh. The groundwater fluctuation in Bangladesh was reported to $20 \%$ and $25 \%$ in 1992 and 1994, respectively and the forecast shows that the fluctuation has reached about $54 \%$ areas of Bangladesh [6]. Although some other countries such as the U.S., Taiwan, Mongolia, Mexico , Argentina, India, and Chile are also facing increasing risk of arsenic contamination in drinking water, the risk faced by Bangladesh by far surpasses that of the other countries [5].To make the matter worse, Bangladesh does not have the necessary technological expertise and economic ability to deal with this risk. Thus, the help of the international community is greatly needed. This paper takes a look at the impacts of arsenic on human and ecological health and subsequent treatments of arsenic related diseases as well as mitigation strategies.

\section{ARSENIC IN GROUNDWATER AND MECHANISM OF CONTAMINATION}

Inorganic arsenic contamination in groundwater poses serious threats in Bangladesh primarily because the major source of drinking water in Bangladesh is shallow tubewells (STWs) [7-8]. More than 30 million people in Bangladesh depend on the tube wells for drinking water [8]. Arsenic contamination in groundwater can occur naturally or industrially. However, in the case of Bangladesh, natural contamination is usually cited as the main source. The arsenic complexes in the soils are chiefly responsible for the natural occurrence of arsenic in the soil. Some condition can lead to the liberation of arsenic from these complexes. Arsenic contamination occurs as soon as it is liberated from complexes owing to the mobility of the ions. The high arsenic contamination in Bangladesh groundwater is encouraged by the alluvial and deltaic sediments which contain pyrite $[4,8]$.

Arsenic contamination in groundwater can occur via oxidation or oxyhydroxide reduction. The oxidation theory proposes that oxidation causes arsenic to be released from the arseno-pyrite in the shallow aquifer. This release is orchestrated by the over exploitation of the groundwater 
which has caused rapid diffusion of oxygen in the pore sediment space. In addition, oxidation occurs due to an increase in dissolved oxygen in the upper layers of the groundwater. Arseno-pyrite is oxidized by the introduced oxygen, forming pitticite, a hydrated iron arsenate, in the presence of water. Pitticite is soft and can dissolve in water. Subsequently, this compound is easily broken into fine particles by the light pressure exerted by tube-well; making it to dissolve in water. It then passes slowly into the water table from the subsoil. According to the reduction theory, desorption from ferric hydroxide minerals under reduction produces arsenic. In this context, the oxidation theory is widely accepted as the primarily means in which arsenic contaminates groundwater in Bangladesh, although both theories have not been validated completely due to lack of enough hydrological and geochemical data for groundwater arsenic pollution in Bangladesh [6].

Arsenic behaves differently in flooded and non-flooded soil conditions. Flooded regions are considered more hazardous than non-flooded regions due to the toxicity and uptake by plants. Moreover, Arsenate (AsV) and arsenite (AsIII) are the most important inorganic forms of arsenic in the soil. AsV is the predominant specie of arsenic in aerobic or oxidizing condition while AsIII is common in anaerobic or reducing condition $[6,8]$.

\section{EXTENT OF ARSENIC CONTAMINATION IN BANGLADESH}

Subsequent to the initial detection of arsenic exposure in Bangladesh, several testing has been carried out to validate the existence and level of arsenic in Bangladesh. Tests were carried out by the Department of Occupational and Environmental Health of the National Institute of Preventive and Social Medicine in 1994 to ascertain arsenic level in the country. Institutions that provided the results include the following: Department of Public Health Engineering's laboratories in the districts of Khulna and Rajshahi, the Jadavpur University in Calcutta, India, the National Institute of Preventive and Social Medicine in Dhaka and the Bangladesh Atomic Energy Commission. The report presented and analyzed a total of 400 sampling sites and several laboratories measured contamination of some wells. The results show more than $50 \mu \mathrm{g} / \mathrm{L}$ concentration of arsenic in about half of the examined concentrations which exceeds the WHO recommended limit as well as the Bangladesh permissible limit $[6,9,10]$. Furthermore, the British Geological Survey (BGS) conducted a survey in which 2022 samples of water were collected from 41 Bangladesh districts affected by arsenic and laboratory tests were carried out for these water samples which show that up to $35 \%$ of the water samples had concentration beyond the Bangladesh permissible limit. The group also reported that up to 21 million people in Bangladesh are exposed to arsenic concentrations beyond the Bangladesh permissible limit in 1998. Based on WHO standard, this number would have been doubled. From August 1995 to February 2000, the School of Environmental Studies and Dhaka Community Hospital collectively analyzed up to 22003 samples of water from tube-wells in about 64 districts of Bangladesh. The study carried out over the course of 5 years indicates that arsenic concentration is beyond $0.01 \mathrm{mg} / \mathrm{L}$ (the WHO recommended limit) in 54 of the 64 districts and beyond $0.05 \mathrm{mg} / \mathrm{L}$ (the Bangladesh permissible limit) in 47 of the 64 districts. Furthermore, the Dhaka Community Hospital in conjunction with the School of Environmental Studies also carried out a field survey in Samta village (district Jessore) between September 1996 and June 1997. About 265 tube-wells were involved in the study, and the study indicated that up to $91 \%$ of the 265 (corresponding to about 241) tube-wells had a concentration of arsenic beyond the Bangladesh permissible limit of $0.05 \mathrm{mg} / \mathrm{L}$. A project authorized by the government of Bangladesh in 1997 to determine the extent of the problem involved the survey of two hundred villages, which were already identified to have arsenic contaminated tube-wells, were surveyed by the Rapid Action Program. The villages have a total population of 469,424 and of the 32, 651 tube-wells that were sampled in these villages, $62 \%$ had a concentration of arsenic greater than $100 \mu \mathrm{g} / \mathrm{L}$. Moreover, the highest level of arsenic contamination $(14 \mathrm{mg} / \mathrm{L})$ was found by experts from the Bangladesh Council for Scientific and Industrial Research (abbreviated as BCSIR) in shallow tube-wells located at Pabna. These statistics also show that groundwater in $85 \%$ of the total area of Bangladesh has been contaminated by arsenic [6,9].

Moreover, data available on arsenic exposure in Bangladesh show that up to 75 million folks in the country are at potential risk of arsenic contamination (Safiuddin and Karim, 3). On the other hand, the total population in regions where some wells are known to be contaminated in Bangladesh is between 35 and 77 million (Smith et al. 1096). According to Uddin and Huda, among the country's 7-11 million hand pumped sources, about half have been estimated to contain an arsenic concentration that exceeds the Bangladesh permissible limit of $50 \mathrm{ppb}$ (Parts per billion) [5]. Bangladesh has about 8.6 million tube-wells, and $55 \%$ (corresponding to 4.76 million) of these tubewells have been tested of arsenic and up to 3.3 million $(39 \%)$ are known to be safe whereas 1.4 million (16\%) are noted to contain arsenic in an amount that is unsafe for human consumption. Up to 20 million people in Bangladesh use tube-wells that contain arsenic beyond the permissible level [5].

\section{IMPACTS OF ARSENIC 4.1 Effects of Arsenic on Crops}

The relationship between arsenic crop yield and irrigation water has not been widely studied and therefore, insufficient data are available in this regard. However, an increase in arsenic concentration in the soil may be able to cause a reduction in the quality of the soil and also lower crop yield [10-11]. The risk on crop production cannot be completely assessed at the moment because of the limited data available in the field. Rice is one of the common foods eaten in Bangladesh and several other Asian countries. Heikens pointed out that rice contributes significantly to the daily uptake of arsenic in Bangladesh [8]. Most of the irrigation water in Bangladesh is contaminated with arsenic, and due to prolonged irrigation with contaminated 
water, food chain contamination can occur. A predominant effect of arsenic accumulation in the soil is land degradation. It is expected that land degradation will increase as arsenic accumulation in soil increases. Thus, it is vitally important to minimize and prevent the input of arsenic in the soil. Management option that could be leveraged to minimize the concentration of arsenic in the soil includes using more irrigation water than necessity, breeding crops tolerant to arsenic, shifting from rice to crops that demand less water especially in the dry season and so forth [8].

Moreover, Alam and Islam conducted a study to understand the negative effects of arsenic contamination on crop production [1]. The study was carried out in three selected regions of Bangladesh prone to arsenic contamination including Kachua, Bhanga, and Faridpur. Primary data was obtained from the samples collected and several variables such as the productivity variability, Cobbdouglas yield function and the factors that influence productivity were tested. From the analysis, it was obvious that of the households sampled, $70 \%$ faced arsenic-related problems in rice production. Arsenic contamination led to uneven plant problems, red coloration of rice plants and canal, un-uniform maturity, unfilled grain, shorter plants, less tillering and reduction in plant yield. Furthermore, land degradation occurred as a result of the continuous use of irrigation water contaminated with arsenic. Fertilizers application and water drainage from the rice fields were employed to overcome some of the problems associated with arsenic contamination but the application of additional fertilizer and labor increased the cost of production of the Boro rice and thereby making the cost of rice production in arsenic contaminated fields $5 \%$ higher than the cost in less contaminated fields. The lower gross return was also recorded as a result of the reduced yield (about $40-50 \%$ lower when compared to less arsenic contaminated plots) of modern variety of boro rice in arsenic contaminated plots. Most farmers have realized that the health of the soil has greatly reduced as a result of arsenic contamination. This poor soil health has been the major reason for low soil productivity. No improvement was observed in plant yield even though farmers made use of fertilizers. Subsequently, this shows that the use of arsenic contaminated irrigation water has caused remarkable degradation of the agricultural land. Furthermore, a decreasing relationship was observed between irrigation of the Boro fields and the yield of the Boro rice [10,12, 13,].

\subsection{Health Effects of Arsenic}

Arsenic health effects do not appear spontaneously, and they take a long time after ingesting drinking water contaminated by arsenic to manifest. Thus, development of arsenic related diseases involves the past and continuous exposure to arsenic. Usually, the first recognized health effects for people exposed to arsenic is skin lesions which include diffuse melanosis accompanied by spotted melanosis, hyperpigmentation, and keratosis. In the case of Bangladesh with a large number of tube wells installed over the course of 5 to 20 years ago, arsenic contamination occurs for people that will and have continued to drink of water obtained from contaminated tube wells over the course of the year $[3,9,14]$. Earlier reports on the health effects caused by arsenic date back to 100 years ago and include some [ dermatological effects such as alterations in pigmentation, hyperkeratosis, skin cancer and so forth. Cases of lung cancer caused by arsenic exposure were identified in the 1940s [15]. Multisystem adverse health effects can occur when arsenic is chronically ingested. When consumed in excess amount, arsenic can cause varieties of health effects including skin manifestation, vascular diseases (such as arteriosclerosis, peripheral vascular disease, ischemic heart disease (ISHD), renal disease, neurological effects, chronic lung disease, cardiovascular diseases, reproductive effects, cerebrovascular disease, and cancers of the skin, lungs, kidney, bladder and liver. Diabetes that does not depend on insulin shortage can also occur on increased exposure to arsenic. The reduced intellectual function in some Bangladesh children is caused by arsenic exposure from drinking water. Growth retardation in children, spontaneous abortion, infant mortality and morbidity, fetal loss, small size at birth and stillbirth are some of the effects of arsenic exposure [4,5,15],

\subsubsection{Carcinogenicity of Arsenic}

Arsenic is a widely known carcinogenic and toxic element. It is one of the reported chemicals known to cause cancer. It was observed far back in the 1800 s as a primary cause of cancer, and in the 1930s, evidence shows that arsenic causes skin cancer. Arsenic was also noted to cause internal cancer especially in the urinary tracks and lung based on evidence in the 1960s [3].

The permissible limit of arsenic in Bangladesh is already noted to be $50 \mu \mathrm{g} / \mathrm{L}$, however, reports released by the National Research Council indicated that the risk of cancer might be in the order of 1 in $100(1 \%)$ for arsenic concentration at this permissible limit. Although studies on the carcinogenicity of arsenic are usually targeted to countries like Taiwan, Argentina, and Chile but similar results can be applied to Bangladesh. In the case of exposure of 29 million people to arsenic above the permissible limit in Bangladesh, then between 200,000 and 300,000 arsenic-related cancer diseases may be recorded if exposure to the chemical is not reduced. Moreover, the poorer nutritional status in Bangladesh would even exacerbate this risk than the estimate given above. The specific estimate of current arsenic-related cancer diseases is not known, but various cases show that many people in Bangladesh are at potential risk of cancer as a result of arsenic ingestion and if exposure is not reduced, many more cases would likely occur. Thus, it is expected that as soon as the latency period is passed, there would be a remarkable increase in mortality caused by internal cancer. To make the matter worse, it is unlikely that both the physician and the individual would understand that the cancer is caused by arsenic and therefore the only way to detect the increase would be through epidemiological studies [3].

\subsubsection{Effects on the Skin}

There are several skin manifestations caused by exposure to arsenic. Few cases of skin cancer have already 
started to appear in Bangladesh due to the fact that the latency of the cancer is about 20 years. This evidence shows that there would be many cases of such skin cancer in the near future in Bangladesh. Moreover, Keratosis (an arsenic caused skin cancer) has a latency of about 10 years. Skin lesion can also occur in children having age less than 10 years, but usually, skin lesions appear 10 years after exposure to the chemical. Keratosis is not the only skin manifestation caused by exposure to arsenic. Other manifestations include characteristic melanosis, basal cell carcinoma, and squamous cell carcinoma. If both keratosis and melanosis are present, chronic arsenic toxicity is confirmed. Melanosis could be found on the trunk and externalities or the entire part of the body. Different forms of melanosis exist, and these include spotted melanosis and diffuse melanosis, also called spotted pigmentation and hyperpigmentation, respectively, leucomelanosis and nonmelanosis also called depigmentation as wel. Hypopigmentation can cause characteristic rain drop pattern. Keratosis often appears on the sole and palm that can be observed in Figure 1 and 2. In this form, it could be keratosis or discrete/nodular keratosis. It could also occur as a combination of spotted and nodular keratosis; it is known as spotted palmoplanter keratosis in this case. Depigmentation is also arsenic caused skin lesion that can result to basal cell carcinoma and Bowen's disease [4]. Safiuddin and Karim pointed out that data collected by governmental bodies, NGOs, and private organizations showed that a lot of people in Bangladesh are suffering from melanosis, keratosis, leuco-melanosis, hyperkeratosis, dorsum, non-petting oedema, gangrene and skin cancer. Melanosis has the highest prevalence $(93.5 \%)$, followed by Keratosis $(68.3 \%)$ and then Leuco-melanosis $(39.1 \%)$ and hyperkeratosis (37.6\%) [6]. Skin cancer has the lowest prevalence in Bangladesh $(0.8 \%)$, and it is often identified among people that have been seriously affected by arsenite and arsenate. Studies conducted by the School of Environmental Studies and Dhaka Community Hospital which involved the analysis of 11000 hairs, nail, urine and skin-scale samples of affected people in Bangladesh showed that more than $90 \%$ of the people have urine, nail and hair above the normal level. Normal arsenic concentration in the hair is between 0.08 and $0.25(\mathrm{mg} / \mathrm{Kg})$, but a concentration of $1 \mathrm{mg} / \mathrm{kg}$ in the hair shows toxicity [6].

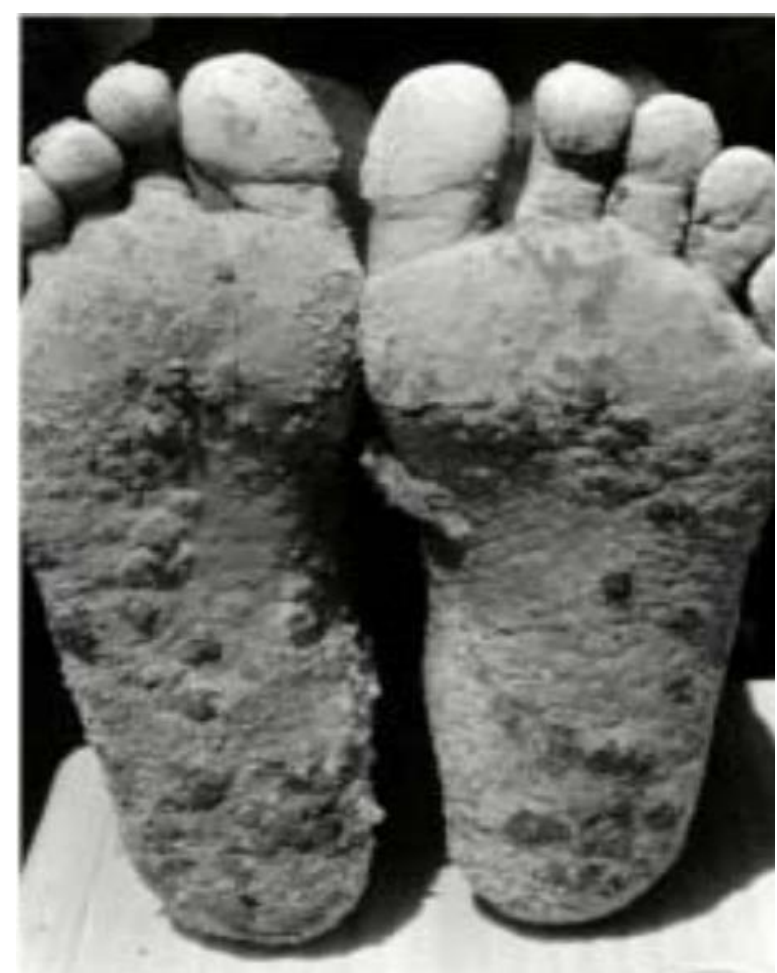

Figure 1: Arsenic related skin lesions on soles [6]

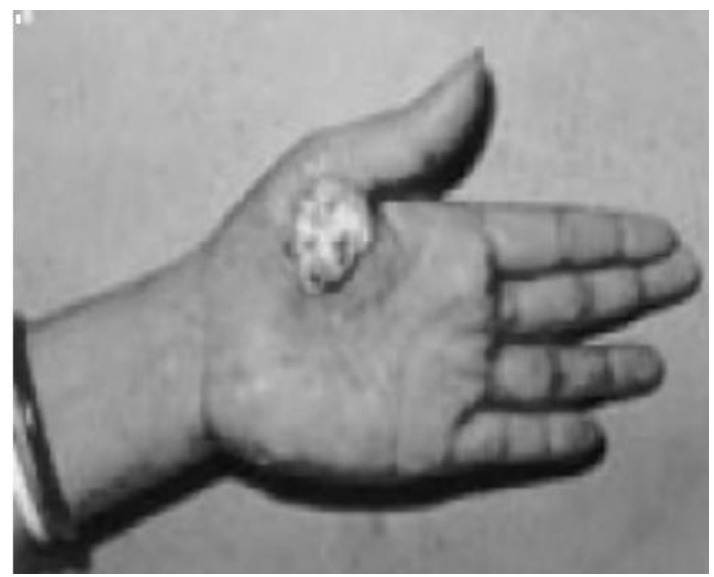

Figure 2: Arsenic related skin lesions on palm [6]

\subsubsection{Effects on the Nervous System}

The toxic effects of heaviest metals such as arsenic, mercury and lead mostly target the central nervous system. Chronic exposure to arsenic contaminated drinking water can cause adverse effects on the nervous system and can lead to reversible peripheral neurological damage. Longterm exposure to inorganic arsenic can lead to peripheral neuropathy. Exposure to arsenic can also cause confusion, disorientation, behavioral changes, cognitive impairment and loss of memory. Cerebrovascular diseases, particularly cerebral infarction, are prevalent due to the chronic ingestion of arsenic contaminated water. Several studies have investigated the prevalence of chronic arsenic ingestion and the resultant neurological diseases in countries like the U.S, Japan, Czechoslovakia, and China. In some of these countries, the primary means of exposures include occupational exposure, arsenic contaminated 
drinking water, living near power plants burning coal for fuel and so forth. Exposed persons in the studies had several neurological diseases including pressure and temperature sensation abatement, abnormal distal sensation, and vegetative nerve functional lesions such as the absence of perspiration as well as diminished perspiration when compared to a normal person [4].

\subsubsection{Effects on the Lung and the Respiratory System}

Arsenic also exists in the form of airborne particles particularly arsenic trioxide has been shown to affect the respiratory system, especially in industrial areas. When exposed to arsenic through routes other than inhalation, the respiratory system could be greatly affected, and it can also result to a chronic cough and bronchopulmonary diseases. It is found that shortness of breath, the intensity of cough, and chest sound in the lungs of both males and females increases with age and concentration of arsenic in water in Bangladesh and West Bengal [4,16,17].

\subsection{Social Impacts}

Arsenic-related diseases are considered contagious or a curse in Bangladesh. Thus, people suffering from arsenic related diseases in Bangladesh also suffer social stigma [5]. People suffering from arsenic diseases in the villages are usually isolated by their neighbors, friends, and relatives. They are avoided and dismissed from public meetings and offices. Children affected by the diseases are banned from attending schools and people are refused jobs, even though they are qualified for them, simply because they are suffering from arsenic diseases. Women face the brunt of the social stigma. The discrimination and ostracization of women suffering from arsenic arsenicosis is truly alarming. Young ladies suffering from the diseases are often made not to marry, and married women suffering from the diseases are deemed unfit to be wives and sent back to their parents with their children $[6,10]$.

\subsection{Economic Implications}

Arsenic-related diseases and loss have grave economic implication on Bangladesh. Flanagan et al (2012) estimated the economic losses that result from arsenic-related death burden in Bangladesh by calculating the productivity loss in relation to per capita GDP [7]. In 2009, IMF estimated that the per capita GDP for Bangladesh was 1465 purchasing power equivalence dollars. On assuming steady economic growth and an average loss of 10 years of productivity due to arsenic-caused death, a loss of USD 12.5 billion can occur over the next 20 years due to arsenic related deaths. This is because of consistency in exposure as in 2009 , which is above $10 \mu \mathrm{g} / \mathrm{L}$. The great economic loss is basically because in Bangladesh, people spend a greater proportion of time working and therefore any loss of life in Bangladesh would result to a greater reduction in productivity than some other countries like the United States. Further, the economic burden is even further underestimated because the estimate does not consider health costs and other costs on the society. Also, the effect would even be more if the country develops and the life expectancy of the people increases. With the increase in medical cost to prolong the lives of people with arsenic related diseases, morbidity burden will likewise increase and therefore increasing the resultant economic loss [7].

\section{RECCOMMENDED REMEDIAL MEASURES}

The first priority in treating arsenicosis is supplying the patient with drinking water free from arsenic. If some other effective treatment methods are not available, it is recommended that the patient is continually given arsenicfree water until the condition abates. Furthermore, the arsenic patient should be monitored in order to make sure that the patient remains unexposed of the chemical. The risk of further contamination of arsenic is greatly reduced if the patient is given arsenic free water. However, no welldesigned study has been carried out in order to show that an improvement in skin keratoses would be observed on ceasing exposure to arsenic. Nonetheless, interview with patients indicates that improvements are observed in the case of mild and moderate keratosis $[7,15]$.

\subsection{Chelation therapy}

In Bangladesh, chelation therapy is commonly administered to arsenic patients to treat arsenicosis [9]. Chelation therapy provides a chemical that binds strongly with arsenic and then can be excreted through urination. This treatment method is efficacious in removing a large amount of arsenic from the body in just a few hours. However, there are few problems associated with chelation especially in the case of chronic arsenic exposure. Firstly, in the case of chronic arsenic exposure, arsenic is excreted from the body even when chelation therapy has not been applied. Chelation can remove arsenic bound to the skin but since arsenic may take many years to accumulate, chelation therapy may make little difference and may not reduce the future risk of cancer. Furthermore, it is not known whether chelation can improve keratoses more rapidly than stopping exposure would. Secondly, there is no clinical trial that provides evidence of the effective of the chelation therapy. Cessation of exposure to arsenic leads to improvement in skin lesions, thus it is undetermined whether improvement usually attributed to chelation occurs due to chelation or cessation. Thirdly, if the patients continue to drink contaminated water, chelation therapy may not produce any positive result, and the condition may even deteriorate remarkably. It is therefore obligatory that careful and controlled study on this therapy be conducted, especially in patients with keratoses and other arsenic related diseases, so as to determine its validity $[9,16]$.

\subsection{Nutrition}

One of the most imperative factors that trigger arsenic related diseases in Bangladesh is malnutrition. Evidently, about $80 \%$ of people in Bangladesh do not have sufficient nutrients, and some suffer from chronic hunger [6]. There persists a high prevalence of micronutrient deficiency in Bangladesh, especially among poor children and women. Vitamin A and iron deficiencies are the most well-known deficiencies. It should be noted that the deficiency of important nutrients makes people more sensitive to diseases and toxicants, including arsenicosis [8]. From 
evidence collected in Taiwan, it can be perceived that nutritional factors can actually adjust the cancer risks associated with arsenic. Health conditions can be improved significantly by providing vitamins and improving the nutrition of patients. Thus, in treating patients suffering from arsenicosis, it is recommended that they should be given multivitamin tablets.

\subsection{Other Possible Treatment Methods}

The advances stage of keratosis could be responsible for infecting hands and soles of feet with fungal infections and consequently, can result to serious health problems. A part of the routine care for advanced keratosis may include the provision of moisturizing lotions and treatment for infections. Furthermore, since arsenic can also result to diabetes, it is necessary to conduct urinary glucose tests in patients suffering from arsenicosis and adequate treatment should follow consequently. Treatment and monitoring should be started if necessary following the diagnosis and tests conducted. Furthermore, arsenic can also cause hypertension and therefore it is necessary to monitor the blood pressure of the patient treated of arsenicosis [9]. In the case of vascular diseases, neurological diseases and some other conditions caused by the uptake of arsenic, it may be necessary also to treat the specific condition as was as treating the arsenicosis. In every case of arsenic treatment, it is mandatory that the individual ceases from taking contaminated water because the condition may not improve if contaminated water or contaminated food is continually taken. Alongside with taking non-contaminated water, it is essential that the individual continually takes arsenic-free water. This is evidently the first priority in treating arsenic, and it is recommended no matter the treatment method that has been adopted so as to ensure the best result.

\subsection{Remedying the Situation and Reducing Exposure}

The best plan of action to deal with arsenic in Bangladesh is to reduce exposure. Prevention in this regard can pay off the great dividend. Exposure reduction could be short term solutions, but they can really be leveraged to reduce arsenicosis. One of the principal things to do for reducing exposure would be to identify nearby tube-wells containing water with low arsenic content. Of course, several studies have already identified wells containing a high and low concentration of arsenic in Bangladesh. Secondly, water filter has to be provided to each home. A candle filtration system may easily be leveraged because it is easy to maintain and use but the arsenic-loaded candles have to be disposed of. Moreover, attachments to tubewells designed to remove arsenic from water are already being tested and could be an effective short-term option for removing arsenic. Thirdly, chemical material required should be provided to remove arsenic from household drinking water. The chemicals should be mixed in water containing arsenic and left overnight. The major benefit of such chemicals is that they are cheap and can be easily transported. However, the problem persists due to the utilization of these chemicals almost on a daily basis and also the sludge has to be disposed of after each treatment. Also, some people may not feel comfortable to use the chemicals on a daily basis. Fourthly, drinking water for the household should be sourced from surface water that has been treated for chlorination and filtration so as to avoid the risk of contaminating with further impurities from the water sources. Lastly, on identifying temporary water sources, it is necessary to close wells that have been highly contaminated with arsenic. Although these interventions are not expensive with respect to the materials concerned, they are expensive as per training, education, and monitoring. It is not advisable to treat the water with alum or to just allow it to stand in order to permit the arsenic to settle because these methods may only reduce arsenic in the water by $50 \%$ as against the recommended reduction by $80-90 \%$. Therefore, the cumulative dose on which arsenic related diseases are based upon may not be altered [9].

Arsenic can also be removed from drinking water by oxidation with oxidants like free chlorine, ozone, permanganate, hypo-chlorite, Fenton reagent $(\mathrm{H} 2 \mathrm{O} 2 / \mathrm{Fe} 2+)$ and so forth. Deep aquifers can be resorted in order to extract potable drinking water free from arsenic. Efficient water supply system can be adopted in Bangladesh to provide safe drinking water and curb the risks associated with arsenic contamination. This system should be used in providing water in the entire country, but most especially in regions whose tube-wells are already known to contain an excess concentration of arsenic. Furthermore, the development of efficient sewage and waste disposal system can also go a long way in reducing arsenic contamination in Bangladesh. This is important because arsenic can contaminate the soil and water supplies through inefficient sewage and waste disposal and thus making it efficient would go a long way in solving this problem. In addition, the restoration of natural river flow and groundwater level can greatly solve the problem. Since studies have unveiled that arsenic contamination in groundwater occurs due to excessive exploitation of these water sources, restoration of the natural groundwater to its level prior to 1975 can greatly reduce the problem. Of course, the restoration will not completely flush arsenic contamination of the water sources in a flicker of seconds, but it will dilute the concentration and even make it low enough to be within the recommended limit. This can greatly reduce the severity of arsenic contamination and make water available for irrigation, drinking and also the industry $[7,18]$. When choosing arsenic mitigation strategies, it is very vital to consider social acceptability and sustainability in addition to costs of the technologies that may be involved).

\section{CONCLUSION}

Arsenic contamination is a serious problem in Bangladesh. Over exploitation of the groundwater coupled with the alluvial and deltaic sediments containing pyrite leads to excessive accumulation of arsenic in the groundwater. The WHO recommended limit of arsenic concentration in ground water is $10 \mu \mathrm{g} / \mathrm{L}$ while Bangladesh permissible limit for arsenic is $50 \mu \mathrm{g} / \mathrm{L}$. However, several tube-wells in Bangladesh have been found to contain arsenic in concentration that is beyond the permissible limit. Up to 75 million people in Bangladesh are at risk of arsenic exposure. Arsenic affects crops and the food chain 
and can result to poor yield of crops. Diseases associated with arsenic include several kinds of cancer, neurological diseases, and skin manifestations including skin lesions, vascular diseases, diabetes and so forth. Treatment is possible through appropriate nutrition and providing arsenic-free drinking water to patients. Some advanced technologies can also be leveraged to mitigate arsenic contamination. Remediation methods can be resorted to but most important would be to close down wells that have a concentration of arsenic beyond the permissible limit. Although the government of Bangladesh has played a significant role in minimizing or eliminating arsenic contamination, it is not capable enough to deal with the problem. The help of the international community is greatly needed to mitigate these effects and delaying action can give a chance for worse conditions. Speedy intervention is required to prevent the issue from escalating.

\section{REFERENCES}

[1] M. Alam and M. Islam, "Assessing the effect of arsenic contamination on modern rice production: evidences from a farm level study", Bangladesh J. Agric. Econs, vol., no. 1, pp. 15-28, 2011. [Accessed 28 November 2016].

[2] N. Ferdousi and S. Huq, "Arsenic Mitigation Approach in Soil by Some Indigenous Sources of Biochar Made at Low Pyrolysis Temperature", International Journal of Plant \& Soil Science, pp. 93-108, 2020. Available: 10.9734/ijpss/2020/v32i930330.

[3] M. Rahman, "Arsenic and Contamination of Drinking-water in Bangladesh: A Public-health Perspective", J Health Popul Nutr, vol. 20, no. 3, pp. 193-197, 2002. [Accessed 28 November 2016].

[4] N. Singh, D. Kumar and A. Sahu, "Arsenic in the environment: Effects on human health and possible prevention", Journal of Environmental Biology, vol. 28, no. 2, pp. 359-365, 2007. [Accessed 28 November 2016].

[5] R. Uddin and N. Huda, "Arsenic Poisoning in Bangladesh", Oman Medical Journal, vol. 26, no. 3, p. 207, 2011. [Accessed 28 November 2016].

[6] M. Safiuddin and M. Karim, "Groundwater arsenic contamination in Bangladesh: Causes, Effects and Remediation", in 1st IEB international conference, Chittagong, 2001, pp. 1-14.

[7] S. Flanagan, R. Johnston and Y. Zheng, "Arsenic in tube well water in Bangladesh: health and economic impacts and implications for arsenic mitigation", Who.int, 2012. [Online]. Available: $\quad$ http://www.who.int/bulletin/volumes/90/11/11101253/en/. [Accessed: 28- Nov- 2016].

[8] A. Heikens, Arsenic contamination of irrigation water, soil and crops in Bangladesh: Risk implications for sustainable agriculture and food safety in Asia, 1st ed. Bangkok: Food and Agriculture Organization of the United Nations Regional Office for Asia and The Pacific, 2006.

[9] A. Allcock, "The Chronic Arsenic Poisoning of the Bangladeshi People", Science $2017 . \quad$ Avends, Available: 10.31988/scitrends.7655.

[10] S. Srivastava and N. Singh, "Mitigation approach of arsenic toxicity in chickpea grown in arsenic amended soil with arsenic tolerant plant growth promoting Acinetobacter sp.", Ecological Engineering, vol. 70, pp. 146-153, 2014. Available: 10.1016/j.ecoleng.2014.05.008.

[11] M. Nassar, "In vitro Mitigation of Arsenic-induced Toxicity by Reduced Glutathione in Rat Pulpal Cells", European Endodontic Journal, 2020. Available: 10.14744/eej.2020.26878.

[12] B. Ahmed, "Arsenic in food chain through irrigation water-soilcrop pathway: Risk assessment for sustainable agriculture of Bangladesh", M.Sc, Swedish University of Agricultural Sciences, 2009.

[13] A. Smith, E. Lingas and M. Rahman, "Contamination of drinking-water by arsenic in Bangladesh: a public health emergency", Bulletin of the World Health Organization, vol. 78, no. 9, pp. 1093-1103, 2000. [Accessed 28 November 2016].
[14] M. Rakib, M. Elahi, M. Akter, M. Hossain, S. Hossain and M. Huda, "Arsenic Poisoning and Social Crisis in Bangladesh", Advances in Research, vol. 5, no. 5, pp. 1-10, 2015. Available: 10.9734/air/2015/19076

[15] S. Loewenberg, "In Bangladesh, arsenic poisoning is a neglected issue", The Lancet, vol. 388, no. 10058, pp. 2336-2337, 2016. Available: 10.1016/s0140-6736(16)32173-0.

[16] C. Hopenhayn, "Arsenic in Drinking Water: Impact on Human Health", Elements, vol. 2, no. 2, pp. 103-107, 2006.

[17] M. Mehmood, A. Shafiq-ur-Rehman, A. Rashid, S. Ganie and R. Bhat, "Environmental Exposure and Hazards of Arsenic: A Review", International Journal of Engineering Research and, vol 6, no. 05, 2017. Available: $10.17577 /$ ijertv6is050186.

[18] K. Man, "Removal of Arsenic from Water Using Chitosan and Nanochitosan", PhD, The Hong Kong University of Science and Technology, 2009. 